\title{
Application of the General Method in the Design of Transmission Poles João SOUSA ${ }^{\mathrm{a}}$, Jorge HENRIQUES ${ }^{\mathrm{b}}$, Rui BARROS ${ }^{\mathrm{c},}$ \\ Faculty of Engineering of the University of Porto, Civil Engng Dept, Structural Division, Portugal \\ ${ }^{a}$ ec08249@fe.up.pt, bjfmh@fe.up.pt, c, ${ }^{*}$ rcb@fe.up.pt \\ ${ }^{*}$ Corresponding author
}

Keywords: General Method, Transmission Poles, Design, Eurocode.

\begin{abstract}
The work aims at designing a transmission pole structure which global security and stability is ensured through the application of the general method for verifying the lateral flexional buckling and lateral torsional buckling of structural components, as present in Eurocode 3 (EC3).
\end{abstract}

\section{Introduction}

The objective of this work was part of the master of science dissertation of the first co-author [1] under supervision of the third co-author, on the comparative analysis of transmission poles executed in mild steel and in high strength steel under the design conditions of Eurocode 3 (EC3) [2].

Before the arrival of computers and automatic calculation methods, norms and regulations only had simplified formulas and procedures due to the severe limitations of manual and personal calculation. In general, the process of structural design consisted of two main phases [3]:

- Analysis - calculation of forces, stresses, etc. for a certain loading and for specified regulatory combinations;

- Resistances - calculation of structural limits, such as cross-sections or connections resistances for a given analysis.

The first step involved the calculation by simple tools whose results were unambiguous and where the more complex structural effects, such as $2^{\text {nd }}$ order effects or torsion, were simplified by approximate values or simply ignored. The second phase, very controlled and governed by norms, was based on simple principles to enable the manual calculation and had in mind the uncertainties associated with the simplification of models. As consequences of these various simplifications, the structural design was developed only at cross-section and individual structural components level. Indeed, the multiple failure and collapse modes associated with the complete structure (impossible to ascertain just at the level of individual structural elements) were not possible to analyze and verify. Over the years the methods of analysis developed considerably but never abandoned this original concept.

In today's modern era, with the emergence of computers and digital revolution, computer programs have established new project boundaries and opened new possibilities for designers, not only because of the speed of calculation but also by the introduction of new numerical methods, which allowed a significant increase in productivity and efficiency. However, the software only influenced positively and qualitatively the analysis phase, with the genesis of new tools for it, but were always limited by structural norms (which indicated and oriented rules based on the manual calculation of individual resistances) in the development of new computational designing tools.

With this issue, the research and development team of structural Eurocodes proposed a first solution: the latest version of EC3-1-1 [2] includes a new general method that enables analysis of resistance and structural stability with full recourse to the potential of structural computing programs, particularly through global 3D geometric models and general finite elements analysis. However, this new method is poorly known and acknowledged by designers due to two main reasons [3]:

- The global structural design philosophy is different from conventional methods;

- The new rules involve greater demands in terms of modeling and structural analysis and these are not yet widely supported by most computer programs used. 


\section{Comparison with the Conventional Method}

The traditional method, most commonly used for stability checking of a steel structure subjected to axial and bending generalized forces, is based on the individual analysis of each structural element and is presented in subchapters 6.3.1, 6.3.2 and 6.3.3 of the EC3-1-1 [2]. This procedure is employed to address situations and problems due to buckling problems (despite the limitations of its application to uniform elements, with simple supports and loads) and is based on two main simplifications [4]:

- The structural element under study is separated from the main structure where it is applied, and on it are applied appropriate equivalent boundary conditions (supports, restraints and loads) during buckling analysis;

- The element analysis is conducted singly for pure buckling modes (pure flexural and pure lateral-torsional buckling) where the interconnection or coupling between them is effected through interaction factors.

However, despite the EC3-1-1 provide parameters for these interaction factors, the definition of the buckling length of each structural element in more complex structures is quite inappropriate in the way that it is only fully resolved or assumed by the designer.

For this conduct, the basic steps for checking the element against buckling are the following:

1) Acquisition of the internal design efforts $\left(\mathrm{N}_{\mathrm{Ed}}\right.$ and $\left.\mathrm{M}_{\mathrm{y}, \mathrm{Ed}}\right)$ in the examined element according to appropriate analysis method (first or second order);

2) Calculation of the critical elastic forces $\left(\mathrm{N}_{\mathrm{cr}}\right.$ and $\left.\mathrm{M}_{\mathrm{y}, \mathrm{cr}}\right)$ of the element according to the appropriate buckling mode - sections 6.3.1.2 and 6.3.2.2 of EC3-1-1;

3) Calculation of the pure ultimate limit forces $\left(\mathrm{N}_{\mathrm{c}, \mathrm{Rk}}\right.$ and $\left.\mathrm{M}_{\mathrm{y}, \mathrm{Rk}}\right)$ of the most critical cross section of the element - equations 6.10 to 6.15 of EC3-1-1;

4) Calculation of the element slenderness $(\lambda)$ and corresponding reduction factors $(\chi)$ for each pure buckling mode:

$$
\begin{aligned}
& \lambda=\sqrt{\frac{N_{c, R k}}{N_{c r}}} \rightarrow \chi(\lambda)(\text { equation } 6.49 \text { of EC3-1-1) } \\
& \lambda_{L T}=\sqrt{\frac{M_{y, R k}}{M_{y, c r}}} \rightarrow \chi_{L T}\left(\lambda_{L T}\right)(\text { equation } 6.56 \text { of EC3-1-1) }
\end{aligned}
$$

5) Acquisition of interaction factors $\left(\mathrm{K}_{\mathrm{zy}}\right)$ between the buckling modes in Annexes A and B of EC3-1-1;

6) Check the element stability through the expressions 6.61 and 6.62 of EC3-1-1.

$$
\frac{N_{E d}}{N_{b, R d}}+K_{z y} \cdot \frac{M_{y, E d}}{M_{b, R d}} \leq 1,0 \text { (equation } 6.62 \text { of EC3-1-1) }
$$

The first step is simple and is commonly performed using models made in structural analysis programs. The third step is easily performed by the formulas given by EC3-1-1 and the remaining steps are straightforward and easy to perform calculations. The second step (calculation of critical elastic forces) is the one that has more influence on the whole process. The EC3-1-1 does not regulate completely its implementation but presents some general requirements. Normally, the calculation is performed through some analytical expressions composed of various parameters related to the support conditions and loading (effective length, gradient moments factor, etc.). Although there is a wide variety of books and articles that help in the determination of these parameters for different types of problems, in general, their choice lies in the designer's knowledge and experience which creates great uncertainty for the project with the possible consequence of an uneconomical and/or unsafe final structure.

This is where the recent general method presents a major change and challenge because it uses the computational calculation capabilities in order to obtain the critical generalized forces. 
The basic concept behind this method is that the analysis is not performed individually on each structural member and for each pure buckling mode, but considering the complete structure, associated with a complex distribution of forces and composition of buckling modes. This allows to obtain solutions in situations where isolation of structural elements does not provide a fully effective, appropriate and correct approach [3]. Therefore, the general method:

- Is applicable not only to single and isolated members but to the complete structural models where the buckling modes are influenced by the structure as a whole;

- Allows the study of irregular elements such as conical components;

- Allows the existence of irregular loads and supports where separation into pure buckling modes is not possible.

Despite being only suitable for checking the buckling of structural elements, their basic application can be extended to other cases.

This method introduces amplification factors to be applied to internal generalized forces in order to achieve the resistance characteristics of the cross sections $\left(\alpha_{\mathrm{ult}, \mathrm{k}}\right)$ and to achieve the buckling resistance of the member in question $\left(\alpha_{\mathrm{cr}}\right)$ :

$$
\begin{array}{ll}
\alpha_{u l t, k, N}=\frac{N_{c, R k}}{N_{E d}} ; & \alpha_{c r, N}=\frac{N_{c r}}{N_{E d}} \\
\alpha_{u l t, k, M}=\frac{M_{y, R k}}{M_{y, E d}} ; & \alpha_{c r, M}=\frac{M_{y, c r}}{M_{y, E d}}
\end{array}
$$

The meaning of these amplification factors is illustrated in Fig. 1:

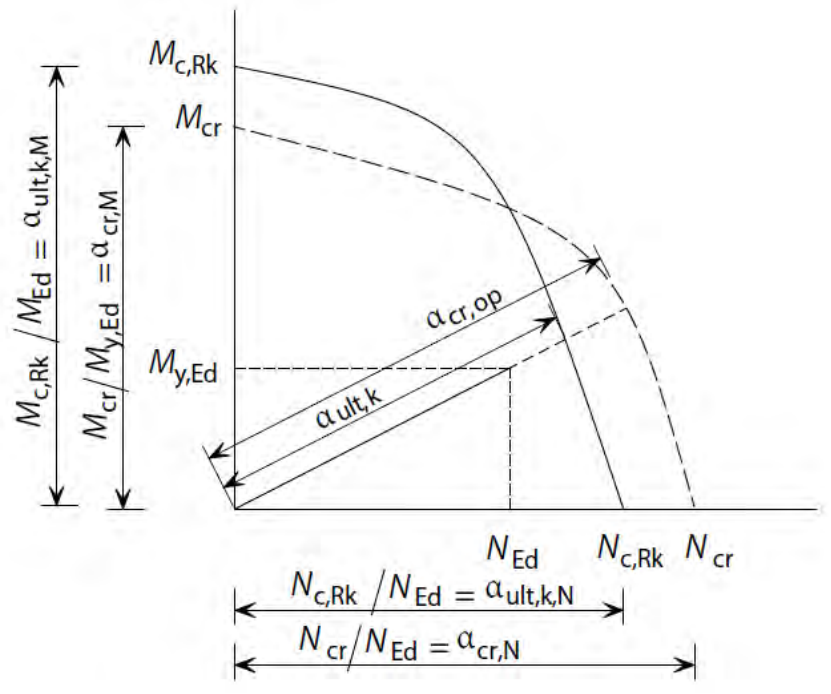

Fig. 1 Load Factors for the General Method [4]

These factors lead to a new way of calculating slenderness and the consequent security checks:

$$
\begin{aligned}
& \lambda=\sqrt{\frac{\alpha_{u l t, k, N}}{\alpha_{c r, N}}} ; \quad 1 \leq \frac{\chi \cdot \alpha_{u l t, k, N}}{\gamma_{M 1}} \\
& \lambda_{L T}=\sqrt{\frac{\alpha_{u l t, k, M}}{\alpha_{c r, M}}} ; \quad 1 \leq \frac{\chi_{L T} \cdot \alpha_{u l t, k, M}}{\gamma_{M 1}}
\end{aligned}
$$

The correct calculation of these factors (which include any kind of global buckling) require, however, more refined analysis and/or specific software. This allows a simple analysis even in cases where the load and the buckling behavior are complex. 


\section{Application}

Transmission poles, given that it is one kind of conical structures whose geometrical cross-sections vary in height, are a typical case of irregular and complex structures for using the traditional method because they are assembled with non-uniform components.

Indeed designing a transmission pole with respective security checks with respect to overall stability, as conducted in the dissertation of Sousa [1], involvs the use of this new general method presented in subchapter 6.3.4 of Eurocode EC3-1-1 [2].

The following steps can divide the process performed:

1) Division of the transmission pole at various points of analysis in height (in a refined form). Sousa [1] defined 94 analysis sections along the full length of the 46.5 meters height post;

2) Acquisition of internal calculation efforts $\left(\mathrm{N}_{\mathrm{Ed}}, \mathrm{M}_{\mathrm{x}, \mathrm{Ed}}, \mathrm{e} \mathrm{M}_{\mathrm{y}, \mathrm{Ed}}\right)$ at each analysis section, and according to the correct type of analysis (first or second order);

3) Calculation of the characteristic resistances of the cross section at each height $\left(\mathrm{N}_{\mathrm{c}, \mathrm{Rk}} \mathrm{e} \mathrm{M}_{\mathrm{Rk}}\right)$ and according to their classification Class (3 or 4), due to the local buckling phenomena;

4) Modelling the structure and respective loads in a finite element program;

5) Calculation of the minimum amplification factor value to be applied to actions in order to reach the elastic critical load $\left(\alpha_{\mathrm{cr}}\right)$, which is achieved through the finite element program (lateral-torsional buckling is rarely conditioning $-\alpha_{\mathrm{cr}, \mathrm{N}}>>\alpha_{\mathrm{cr}, \mathrm{M}}$ ):

$\alpha_{c r}=\min \left(\alpha_{c r, N} ; \alpha_{c r, M}\right)=\alpha_{c r, N}$

6) Calculation of the amplification factor values (9) to be applied to actions in order to achieve the characteristic resistances of each cross section, using the equations found in (4) and (5):

$\alpha_{u l t, k, N} ; \alpha_{u l t, k, M x} ; \alpha_{u l t, k, M y}$

7) Calculation of the minimum amplification factor value to be applied to actions in order to achieve the characteristic resistance of the most critical cross-section of the structure:

$\alpha_{u l t, k}=\min \left(\alpha_{u l t, k, N} ; \alpha_{u l t, k, M x} ; \alpha_{u l t, k, M y}\right)$

8) Calculation of the general slenderness and reduction factors:

$$
\lambda_{o p}=\sqrt{\frac{\alpha_{u l t, k}}{\alpha_{c r}}} \rightarrow \chi\left(\lambda_{o p}\right), \chi_{L T}\left(\lambda_{o p}\right) \quad \text { (equation } 6.64 \text { of EC3-1-1) }
$$

9) Check the transmission pole overall stability with the following equation:

$$
\frac{\chi_{o p} \cdot \alpha_{u l t, k}}{\gamma_{M 1}} \geq 1,0
$$

Where $\chi_{\text {op }}$ is equal to the minimum between $\chi$ e $\chi_{\text {LT }}$.

Even if the last condition (12) is true, it does not exempt other structural safety checks (as shear resistance and torsion resistance, for instance) just like the conventional method.

\section{Examples of inadequacies in the application of the conventional method}

With the application of the traditional method it is necessary to resort to buckling curves and interaction factors during the analysis. These parameters were first calibrated for simple, uniform and simply supported elements. However, this type of situation rarely occurs in real structures of higher 
complexity. It is in this case where some parameters come from the conventional method in order to reduce the actual structure to the model structure so that one can continue the calculations. For the basic buckling modes, these parameters correspond to the effective length factors $\left(v_{\mathrm{y}}, v_{\mathrm{z}}, v_{\mathrm{w}}\right)$ and the moment gradient $\left(\mathrm{C}_{1}, \mathrm{C}_{2}, \mathrm{C}_{3}\right)$ factors.

However, the determination of these parameters is associated with several problems and doubts. According to Papp [3], the most important result from the following considerations:

- In situations where the elements have cantilever-like behavior or have intermediate supports, the solutions to these values are rare and incomplete;

- For the effective torsional length factor $\left(v_{\mathrm{w}}\right)$ there is no practical proposal, even that it can be very influent and dominant in the cases where the buckling mode includes torsion;

- It was just recently realized that the moment gradient factors $\left(\mathrm{C}_{1}, \mathrm{C}_{2}, \mathrm{C}_{3}\right)$ can highly depend on the lateral and torsional effective length factors as well;

- The determination of these factors can be very difficult and uncertain in the cases when the buckling of a certain member is only a part of a global type buckling mode involving a complete part of the structure.

This last point is very important in so far as any structure works as a full system where generally there are some global buckling modes that are not amenable to analysis when it comes to the isolation of the structural members according to the conventional method. It is in this case that the general method constitutes a modernization and upgrading of earlier ones, since it allows improvements in reliability and efficiency during the design process.

Safety checks for buckling usually also include experimental and analytical phases in simple models, designed especially for the analysis of some buckling modes - the regular cases. Any deviation from this scenario originates irregular cases, where the models can be divided into two categories [3]:

- Structural irregularity - corresponds to deviation from the uniform model; for instance: prismatic member model, tapered, haunched and built-up members;

- Behavior irregularity - corresponds to deviation from the examined regular buckling modes: for instance: buckling about an eccentric axis of an element, caused by eccentric lateral supports.

This type of situation is quite common in metal structures and originates problems that the conventional method was not able to solve, and that resulted in a further impulse to the development of the general method.

On the other hand, when using the traditional method for analyzing the critical forces and slenderness for each individual structural element, a common result shows that the values of the member slenderness vary considerably within one coherent structural model. This creates a problem of reliability because of the higher critical forces, and accordingly the lower member slenderness values are calculated with the assumption that the reminder elements of the complete structure are in a stable position. This fact is not true, since in reality their critical forces belong to a lower load level than what could be expected if the elements were isolated. The concept of global stiffness of a structure reflects very well this problem.

It is due to the existence of these cases that the EC1-1-7 [5] suggests, in section 3.3, the following:

- "Designing the structure so that in the event of a localized failure (e.g. failure of a single member) the stability of the whole structure or of a significant part of it would not be endangered"

By employing the general method, these dilemmas are automatically identified and it only yields one critical load factor for all the structural members simultaneously. The robustness of the model structure is not divided or distorted and remains faithful to the real case.

\section{Conclusion}

Using the general method for verifying the structural stability in the design of transmission pole structures [1] has shown to be more efficient as it allows a less expensive and more realistic 
calculation process, mostly because the transmission poles are structures formed by elements with variable cross sections in height. During the analysis, no structural simplifications or modifications were necessary as if it had resorted to the conventional methods of buckling checking.

\section{Acknowledgement}

The authors acknowledge the support from the project "VHSSPOLES - Very High Strength Steel Poles" (Faculty of Engineering of the University of Porto, reference 21518).

\section{References}

[1]Sousa, J.P.P.d., Comparative Analysis of Transmission Poles Executed in Mild Steel and High Strength Steel 2014, Master of Science dissertation (in Portuguese), Faculty of Engineering of the University of Porto: Porto.

[2]EN 1993-1-1: Eurocode 3: Design of steel structures - Part 1-1: General rules for buildings 2010, European Committee for Standardization (CEN): Brussels.

[3]Papp, F. and J. Szalai, New approaches in Eurocode 3-efficient global structural design Part 0: An explanatory introduction.

[4]Szalai, J., The "General Method" of EN 1993-1-1, in New Steel Construction. 2011, The British Constructional Steelwork Association, The Steel Construction Institute, Tata Steel: London.

[5]EN 1991-1-7: Eurocode 1: Actions on structures - Part 1-7: Accidental actions. 2006, European Committee for Standardization (CEN): Brussels. 